\title{
Psychometric Validation of the Sexual Function Questionnaire in Poland
}

\author{
Slawomir Slaski • Malgorzata Stefankiewicz
}

Published online: 11 September 2011

(C) The Author(s) 2011. This article is published with open access at Springerlink.com

\begin{abstract}
Questionnaires diagnosing sexual dysfunctions in women are important tools which can facilitate the diagnosis and therapy of individual female patients. The study is aimed at the adaptation of the Sexual Function Questionnaire, which was designed by Frances H. Quirk and associates for the American population, to Polish conditions (Quirk et al. in J Sex Med 4:469-77, 1). The study group consisted of 143 women aged 20-68 who were receiving gynaecological care and displayed various types of sexual dysfunction (e.g. female sexual arousal disorder, female orgasmic disorder, dyspareunia), or none of those. The good psychometric quality of the Sexual Function Questionnaire has been confirmed with the following results: convergent validity $\mathrm{r}_{\mathrm{s}}=0.62, p<0.01$, construct validity was estimated by Principal Component Analysis with the promax rotation method. The 7 factors together explained $80.7 \%$ of total variance, and a reliability of 0.97 for the whole test, with that for particular domains ranging from 0.62 to 0.96 (estimated with Cronbach's alpha). The obtained results are mostly consistent with results from studies conducted by the authors of the Questionnaire in the United States, and with the adaptation study carried out in Iran. There are, however, some differences in the assignment of questions to particular domains, as well as in the names of the domains.
\end{abstract}

Keywords Female sexual dysfunctions · Adaptation · Questionnaire · Poland

\section{Introduction}

According to studies and experts' opinions, as many as 40-50\% of women may suffer from sexual dysfunctions, but the detectability of such conditions and the percentage of women who receive treatment is rather low. In many cases, the person suffering from sexual

S. Slaski $(\bowtie)$

Institute of Psychology, Cardinal Stefan Wyszynski University, Ul. Woycickiego 1/3 Blok 14, 01-938 Warsaw, Poland

e-mail: s.slaski@uksw.edu.pl

M. Stefankiewicz

Faculty of Psychology, University of Finances and Management, Warsaw, Poland 
dysfunction is not aware of her problem or is incapable of overcoming her embarrassment and seeking professional help. Another problem is the poor availability of sexologists, who usually work in large cities, and generally there are too few of them [1-3].

Self-administered questionnaires would be thus of invaluable help in preliminary diagnosis of sexual dysfunctions. They would make it possible for general practitioners or psychologists without sexological training to diagnose the problem and refer the patient to a specialist for a full diagnosis and therapy, while fully respecting the privacy of the patient.

The main purpose of this article is to describe the procedure for the adaptation of the Sexual Function Questionnaire to the Polish population. The SFQ can both provide additional information about female sexual dysfunctions in our culture and facilitate their diagnosis and therapy.

\section{Methods}

In order to monitor the process of cultural adaptation of the Sexual Function Questionnaire, which primarily diagnoses female sexual dysfunctions, a study has been carried out on a group of Polish women.

The questionnaire was translated from English to Polish by a trained psychologist, then it was back-translated into English by another trained psychologist, and on this basis of the final version was drawn up for the adaptation study [4-6]. The questionnaire consists of 34 questions divided into two sections: Sexual Activity and Sexual Life. The questions concern the last 4 weeks in the life of the persons examined. The Sexual Activity section (27 items) contains questions that involve any activity which may result in sexual stimulation or pleasure such as intercourse, caressing, foreplay, masturbation and oral sex. The Sexual Life section (7 items) consists of questions that involve both physical sexual activity as well as the emotional-sexual relationship with the partner. Questions have been assigned to seven domains (identical as in the original Questionnaire-desire, enjoyment, orgasm, arousal-sensation, arousal-lubrication, pain and partner relationship) based on the way the questions were arranged in the original version of the questionnaire and on the basis of content analysis of the questions (performed by 3 competent judges-persons with psychological and sexological expertise).

\section{Population}

The studied group consisted of 143 women aged 20-68. The average age of the women was 27.65. All the women were gynecological patients from different Polish cities, including 54 who were at the time hospitalized at gynecological, pregnancy pathology and maternity wards (the others were not hospitalized). 44 women were sexological patients suffering from various problems in their sexual life, but without having any sexual dysfunction diagnosed. 45 women were neither hospitalized nor sought sexological treatment.

All the women had already initiated their sexual life, had sexual intercourse, and were heterosexual Caucasians.

The study was carried out in 2009. The criterion for recruitment of participants was the fact of being a gynecological patient. Additionally, for the group of women who were thought to suffer from sexual dysfunction, another criterion was the fact that of receiving sexological therapy. This way of selecting subjects for the study was used for two reasons-patients of a gynecological clinic constituted the study group in the Iranian adaptation study, which was used as the basis for the adaptation procedure in Poland [7]. 
Furthermore, the results obtained from patients of the gynecological clinic made it possible to verify whether the SFQ was capable of detecting sexual dysfunctions well enough for it to be used as a diagnostic tool.

Female subjects took part in the study voluntarily, anonymously and without any remuneration.

\section{Results}

Reliability of the Sexual Function Questionnaire

\section{Internal Consistency}

The reliability of the SFQ measured with Cronbach's alpha test was 0.97 , which proves it is highly reliable.

Due to the reliability results for particular test items, Item 31 was excluded, as it displayed insufficient correlation with the entire questionnaire (0.114). The domain which this item was assigned to by the competent judges (the Partner relationship domain) had its values calculated without this item.

Each item of the SFQ was assigned to one of the 7 domains (identical as in the original SFQ - desire, enjoyment, orgasm, arousal-sensation, arousal-lubrication, pain and partner relationship), based on the assignment pattern to analogical domains in the American validation studies. Based on these results, preliminary domains were constructed for particular variables measured with the SFQ (variables analogical to the names of particular domains), and reliability tests for the domains were carried out.

The reliability of individual scales of the questionnaire measured by Cronbach's alpha ranges varied from 0.62 to 0.96 .

Validity of the Sexual Function Questionnaire

\section{Construct Validity}

The adequacy of the sample for the adaptation study was confirmed by the Kaiser-MeyerOlkin, which was 0.95 at $p<0.001$.

Construct Validity was estimated by Principal Component Analysis with the promax rotation method (Table 1). The 7 factors together explained $80.7 \%$ of total variance.

\section{Convergent Validity}

Convergent Validity of the SFQ was checked with the SFK/K scale (version for females), which examines the quality of sexual life and shows whether or not the subjects suffer from any sexual dysfunction [8].

A non-parametric coefficient was employed for the correlation, which was equal to 0.62 at $p<0.001$. This proves a strong correlation between both questionnaires.

\section{Discussion}

The most serious doubts during the analysis of the reliability of the test items concerned Item 31, which was assigned by competent judges to the Partner relationship domain, and 
Table 1 Factor loadings for the 33 items of SFQ

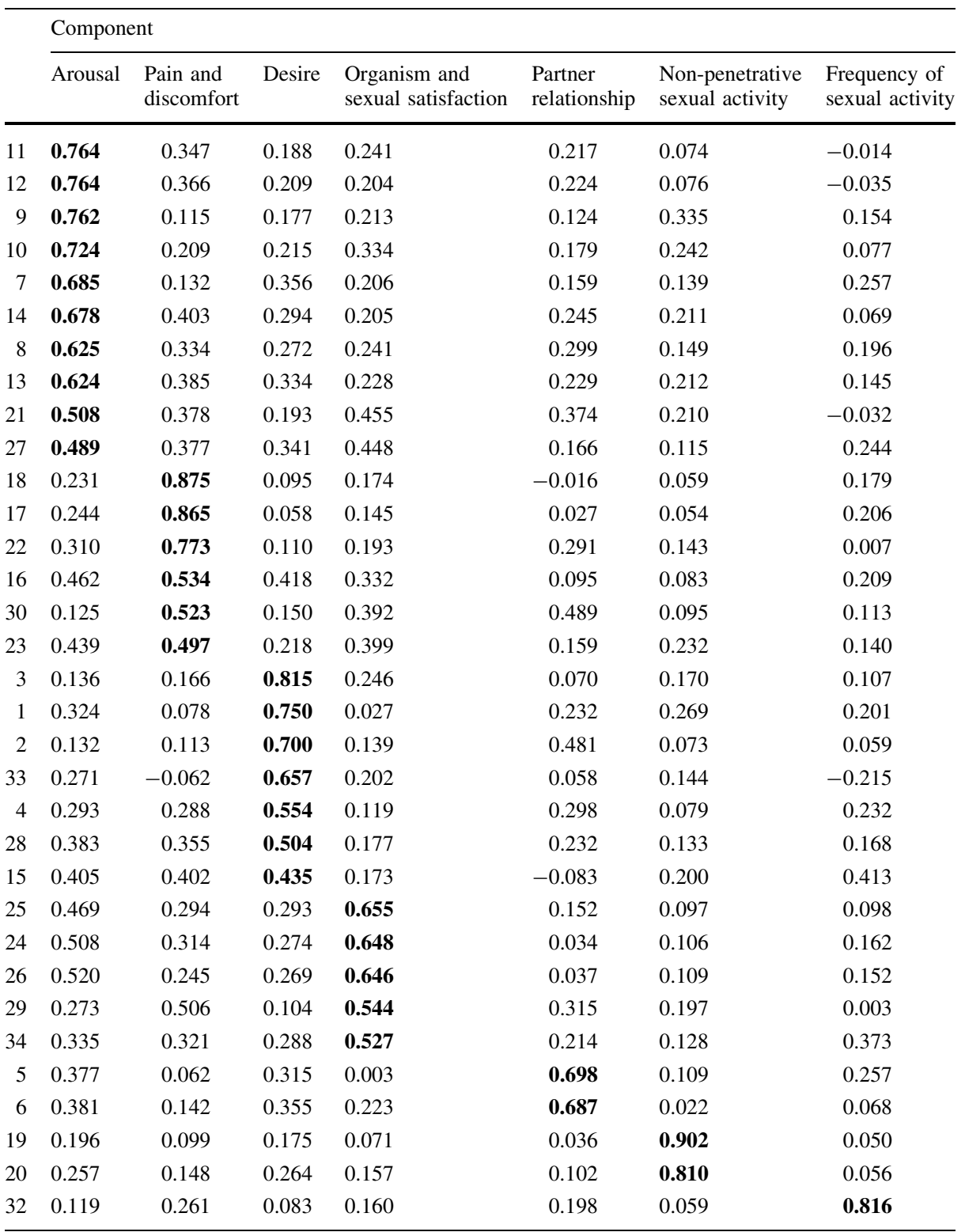

Extraction method: Principal Component Analysis. Rotation method: Promax with Kaiser normalization The bold values represent the item

which read "Thinking about the last 4 weeks, how much did you worry about your partner's negative feelings about your sexual life (e.g., partner feeling angry, hurt or rejected)?". This item scored poorly and thus it would be advisable to remove it from the questionnaire. 
Reliability analysis of the domains also indicates the need to remove not just Item 31 , but the whole domain which it was assigned to, that is, the Partner relationship domain. Its reliability measured with Cronbach's alpha coefficient on the basis of the items being standardized produced the result of 0.62 , which, while indicative of a strong correlation, was the lowest result for all the domains. Additionally, during Cronbach's alpha analysis it was observed that if either of the two items in the domain were removed, the domain would reveal a negative value due to the negative average covariance between the items, which violates the reliability model requirements. As a result of the factor analysis and content reanalysis, it seems advisable to assign the two items (5 and 30) to other domain, e.g desire.

The remaining reliability results for particular domains indicate adequate item-domain assignment and show that the results from the domains very well reflect the variables measured, with negligible error levels.

Construct validity was estimated by means of factor analysis. The result obtained explaining $80.7 \%$ of the total variance can be considered very high.

Convergent validity was estimated by relating the SFQ to the SFK/K scale. The correlation coefficient $r_{s}$ indicates a strong correlation between both questionnaires. This result means that the probability of placing an initial correct preliminary diagnosis on the basis of SFQ is so high that it can be used in the diagnostics of sexual dysfunctions.

Following the results of factor analysis, it seems that the arrangement of questions within the domains should be modified, and some domains should be replaced by others. The suggested arrangement of the items and domains on the basis of factor and content analysis is as follows:

1. Arousal domain: items $7,8,9,10,11,12,13,14,21,27$;

2. Pain and Discomfort domain: items 16, 17, 18, 22, 23, 30;

3. Desire domain: items 1, 2, 3, 4, 15, 28, 33;

4. Orgasm and Sexual Satisfaction domain: items 24, 25, 26, 29, 34;

5. Partner relationship domain: items 5, 6;

6. Non-penetrative Sexual Activity domain: items 19, 20;

7. Frequency of Sexual Activity domain: item 32.

According to content analysis of items, the above arrangement of questions matches the domains well. What is especially interesting is the creation of the sixth domain (Nonpenetrative Sexual Activity). Its validity and adequacy seems to be proven by the results given in Report: Sexuality of Poles 2002 [9]. Its authors pointed out that one in four women displays negative emotions towards masturbation. As Items 19 and 20, which constitute the domain, also concern this type of sexual activity, this may confirm the results for the Polish population. The reliability of this subscale, measured by Cronbach's alpha-test based on standardized items was 0.85 , indicating a very strong relationship. However the reliability of the subscales of Partner and Pain and Discomfort were respectively 0.85 and 0.93 .

Comparing the results of the adaptation study for the Polish population with the validation studies for other countries, the statistical data obtained in Poland seem to be quite similar. Both in Iran and the USA, a shorter version of the test was used, abbreviated as SFQ-V2. It consists of 26 items and 7 domains: Desire, Arousal-sensation, Arousallubrication, Orgasm, Enjoyment, Pain, and Partner relationship). The short version was employed due to the results of the factor analysis following preliminary studies with the longer version. Taking into the consideration the results for the Polish population, such a decision seems to be advisable, and it is also recommended that the shorter version V2 be used in Poland, or new test items be developed on the basis of the SFQ (which would in fact imply the development of a new test). As a result of statistical analysis of the study 
conducted for the American population, the Partner Relationship domain was rejected (just as in Poland), as its statistical values of $0.33-0.65$ was considered to be too low to consider them in further analysis. The reliability of the other domains varied from 0.71 to 0.96 , which is similar to the results obtained in Poland.

Interestingly, in Iran, like in Poland, a non-penetrative sexual activity domain had been formed - the only difference being that it was called the Unusual Sex Domain. In the case of the Polish population, the reasons are explained above. In the case of Iran, the underlying cause may be the religious beliefs of the studied group (Islam forbids this kind of sexual activity, especially among women) as about $72 \%$ of women gave a negative response here. The statistical data obtained from the studies in Iran were estimated as similar to those from the USA.

As the satisfactory adaptation of SFQ study conducted in Poland support the use of this questionnaire in research. It seems that it would be better to use the V2 test, but this would require additional research in order to estimate its psychometric characteristics on a broader population and in different clinical groups.

Acknowledgments The Authors would like to express their gratitude to Tara Symonds, PhD. for her consent to adapt at the Sexual Function Questionnaire to Polish conditions.

Conflict of interest None declared.

Open Access This article is distributed under the terms of the Creative Commons Attribution Noncommercial License which permits any noncommercial use, distribution, and reproduction in any medium, provided the original author(s) and source are credited.

\section{References}

1. Quirk, F.H., Haughie, S., Symonds, T.: The use of the sexual function questionnaire as a screening tool for women with sexual dysfunction. J. Sex. Med. 2, 469-477 (2005)

2. Quirk, F.H., Heiman, J.R., Rosen, R.C., Laan, E., Smith, M.D., Boolell, M.: Development of a sexual function questionnaire for clinical trials of female sexual dysfunction. J. Womens. Health. Gend. Based. Med. 11, 277-289 (2002)

3. Lew-Starowicz, Z., Zaburzenia seksualne w depresjach [Sexual dysfunction in depressions].: Medipress medical update. 2, 11-2 (2005). Available at: http://medipress.pl/pdf/Psychiatria/024.pdf. Accessed 1 May 2009

4. Anastasi, A., Urbina, S.: Testy Psychologiczne [Psychological Tests]. Pracownia Testow Psychologicznych PTP, Warszawa (1999)

5. Brzezinski, J.: Metodologia Badan Psychologicznych. Wybor Tekstow [Methodology of Psychological Research. Selection of texts]. PWN, Warszawa (2004)

6. Zawadzki, B.: Kwestionariusze Osobowosci. Strategie i Procedura Konstruowania [Personality Questionnaires. Strategies and Procedures of Constructing]. Wydawnictwo Naukowe Scholar, Warszawa (2006)

7. Khademi, A., Alleyassin, A., Agha-hosseini, M., Dadras, N., Roodsari, A.A., Tabatabaeefar, L., Amini, M.: Psychometric properties of sexual function questionnaire: evaluation of an Iranian sample. Iran. J. Rep. Med. 1, 23-28 (2006)

8. Kratochvil, S.: Leczenie Zaburzeń Seksualnych [Treatment of Sexual Dysfunction]. Iskry, Warszawa (2002)

9. Lew-Starowicz, Z., Lew-Starowicz, M.: Raport: Seksualność Polaków 2002 [Report: Sexuality of Poles 2002]. Przegl Menop 2002. 4, 64-73 